\title{
Pathologies of Neural Models Make Interpretations Difficult
}

\author{
Shi Feng ${ }^{1}$ Eric Wallace ${ }^{1}$ Alvin Grissom II $^{2}$ Mohit Iyyer $^{3,4}$ \\ Pedro Rodriguez ${ }^{1}$ Jordan Boyd-Graber $^{1}$ \\ ${ }^{1}$ University of Maryland ${ }^{2}$ Ursinus College \\ ${ }^{3}$ UMass Amherst ${ }^{4}$ Allen Institute for Artificial Intelligence \\ \{shifeng, ewallac2, entilzha, jbg\}@umiacs.umd.edu, \\ agrissomeursinus.edu, miyyerdcs.umass.edu
}

\begin{abstract}
One way to interpret neural model predictions is to highlight the most important input features-for example, a heatmap visualization over the words in an input sentence. In existing interpretation methods for NLP, a word's importance is determined by either input perturbation-measuring the decrease in model confidence when that word is removed - or by the gradient with respect to that word. To understand the limitations of these methods, we use input reduction, which iteratively removes the least important word from the input. This exposes pathological behaviors of neural models: the remaining words appear nonsensical to humans and are not the ones determined as important by interpretation methods. As we confirm with human experiments, the reduced examples lack information to support the prediction of any label, but models still make the same predictions with high confidence. To explain these counterintuitive results, we draw connections to adversarial examples and confidence calibration: pathological behaviors reveal difficulties in interpreting neural models trained with maximum likelihood. To mitigate their deficiencies, we fine-tune the models by encouraging high entropy outputs on reduced examples. Fine-tuned models become more interpretable under input reduction without accuracy loss on regular examples.
\end{abstract}

\section{Introduction}

Many interpretation methods for neural networks explain the model's prediction as a counterfactual: how does the prediction change when the input is modified? Adversarial examples (Szegedy et al., 2014; Goodfellow et al., 2015) highlight the instability of neural network predictions by showing how small perturbations to the input dramatically change the output.

$\begin{array}{ll}\text { SQUAD } & \\ \text { Context } & \begin{array}{l}\text { In } 1899, \text { John Jacob Astor IV invested } \\ \$ 100,000 \text { for Tesla to further develop } \\ \text { and produce a new lighting system. In- } \\ \text { stead, Tesla used the money to fund his } \\ \text { Colorado Springs experiments. }\end{array} \\ & \begin{array}{l}\text { What did Tesla spend Astor's money on ? } \\ \text { Original } \\ \text { Reduced } \\ \text { Confidence }\end{array} \\ & 0.78 \rightarrow 0.91\end{array}$

Figure 1: SQUAD example from the validation set. Given the original Context, the model makes the same correct prediction ("Colorado Springs experiments") on the Reduced question as the Original, with even higher confidence. For humans, the reduced question, "did", is nonsensical.

A common, non-adversarial form of model interpretation is feature attribution: features that are crucial for predictions are highlighted in a heatmap. One can measure a feature's importance by input perturbation. Given an input for text classification, a word's importance can be measured by the difference in model confidence before and after that word is removed from the input-the word is important if confidence decreases significantly. This is the leave-one-out method ( $\mathrm{Li}$ et al., 2016b). Gradients can also measure feature importance; for example, a feature is influential to the prediction if its gradient is a large positive value. Both perturbation and gradient-based methods can generate heatmaps, implying that the model's prediction is highly influenced by the highlighted, important words.

Instead, we study how the model's prediction is influenced by the unimportant words. We use input reduction, a process that iteratively removes the unimportant words from the input while maintaining the model's prediction. Intuitively, the words remaining after input reduction should be important for prediction. Moreover, the words 
should match the leave-one-out method's selections, which closely align with human perception (Li et al., 2016b; Murdoch et al., 2018). However, rather than providing explanations of the original prediction, our reduced examples more closely resemble adversarial examples. In Figure 1 , the reduced input is meaningless to a human but retains the same model prediction with high confidence. Gradient-based input reduction exposes pathological model behaviors that contradict what one expects based on existing interpretation methods.

In Section 2, we construct more of these counterintuitive examples by augmenting input reduction with beam search and experiment with three tasks: SQUAD (Rajpurkar et al., 2016) for reading comprehension, SNLI (Bowman et al., 2015) for textual entailment, and VQA (Antol et al., 2015) for visual question answering. Input reduction with beam search consistently reduces the input sentence to very short lengths-often only one or two words-without lowering model confidence on its original prediction. The reduced examples appear nonsensical to humans, which we verify with crowdsourced experiments. In Section 3, we draw connections to adversarial examples and confidence calibration; we explain why the observed pathologies are a consequence of the overconfidence of neural models. This elucidates limitations of interpretation methods that rely on model confidence. In Section 4, we encourage high model uncertainty on reduced examples with entropy regularization. The pathological model behavior under input reduction is mitigated, leading to more reasonable reduced examples.

\section{Input Reduction}

To explain model predictions using a set of important words, we must first define importance. After defining input perturbation and gradient-based approximation, we describe input reduction with these importance metrics. Input reduction drastically shortens inputs without causing the model to change its prediction or significantly decrease its confidence. Crowdsourced experiments confirm that reduced examples appear nonsensical to humans: input reduction uncovers pathological model behaviors.

\subsection{Importance from Input Gradient}

Ribeiro et al. (2016) and Li et al. (2016b) define importance by seeing how confidence changes when a feature is removed; a natural approximation is to use the gradient (Baehrens et al., 2010; Simonyan et al., 2014). We formally define these importance metrics in natural language contexts and introduce the efficient gradient-based approximation. For each word in an input sentence, we measure its importance by the change in the confidence of the original prediction when we remove that word from the sentence. We switch the sign so that when the confidence decreases, the importance value is positive.

Formally, let $\mathbf{x}=\left\langle x_{1}, x_{2}, \ldots x_{n}\right\rangle$ denote the input sentence, $f(y \mid \mathbf{x})$ the predicted probability of label $y$, and $y=\operatorname{argmax}_{y^{\prime}} f\left(y^{\prime} \mid \mathbf{x}\right)$ the original predicted label. The importance is then

$$
g\left(x_{i} \mid \mathbf{x}\right)=f(y \mid \mathbf{x})-f\left(y \mid \mathbf{x}_{-i}\right) .
$$

To calculate the importance of each word in a sentence with $n$ words, we need $n$ forward passes of the model, each time with one of the words left out. This is highly inefficient, especially for longer sentences. Instead, we approximate the importance value with the input gradient. For each word in the sentence, we calculate the dot product of its word embedding and the gradient of the output with respect to the embedding. The importance of $n$ words can thus be computed with a single forward-backward pass. This gradient approximation has been used for various interpretation methods for natural language classification models ( $\mathrm{Li}$ et al., 2016a; Arras et al., 2016); see Ebrahimi et al. (2017) for further details on the derivation. We use this approximation in all our experiments as it selects the same words for removal as an exhaustive search (no approximation).

\subsection{Removing Unimportant Words}

Instead of looking at the words with high importance values-what interpretation methods commonly do-we take a complementary approach and study how the model behaves when the supposedly unimportant words are removed. Intuitively, the important words should remain after the unimportant ones are removed.

Our input reduction process iteratively removes the unimportant words. At each step, we remove the word with the lowest importance value until the model changes its prediction. We experi- 
ment with three popular datasets: SQUAD (Rajpurkar et al., 2016) for reading comprehension, SNLI (Bowman et al., 2015) for textual entailment, and VQA (Antol et al., 2015) for visual question answering. We describe each of these tasks and the model we use below, providing full details in the Supplement.

In SQUAD, each example is a context paragraph and a question. The task is to predict a span in the paragraph as the answer. We reduce only the question while keeping the context paragraph unchanged. The model we use is the DRQA Document Reader (Chen et al., 2017).

In SNLI, each example consists of two sentences: a premise and a hypothesis. The task is to predict one of three relationships: entailment, neutral, or contradiction. We reduce only the hypothesis while keeping the premise unchanged. The model we use is Bilateral Multi-Perspective Matching (BIMPM) (Wang et al., 2017).

In VQA, each example consists of an image and a natural language question. We reduce only the question while keeping the image unchanged. The model we use is Show, Ask, Attend, and Answer (Kazemi and Elqursh, 2017).

During the iterative reduction process, we ensure that the prediction does not change (exact same span for SQUAD); consequently, the model accuracy on the reduced examples is identical to the original. The predicted label is used for input reduction and the ground-truth is never revealed. We use the validation set for all three tasks.

Most reduced inputs are nonsensical to humans (Figure 2) as they lack information for any reasonable human prediction. However, models make confident predictions, at times even more confident than the original.

To find the shortest possible reduced inputs (potentially the most meaningless), we relax the requirement of removing only the least important word and augment input reduction with beam search. We limit the removal to the $k$ least important words, where $k$ is the beam size, and decrease the beam size as the remaining input is shortened. ${ }^{1}$ We empirically select beam size five as it produces comparable results to larger beam sizes with reasonable computation cost. The requirement of maintaining model prediction is unchanged.

\footnotetext{
${ }^{1}$ We set beam size to $\max (1, \min (k, L-3))$ where $k$ is maximum beam size and $L$ is the current length of the input sentence.
}

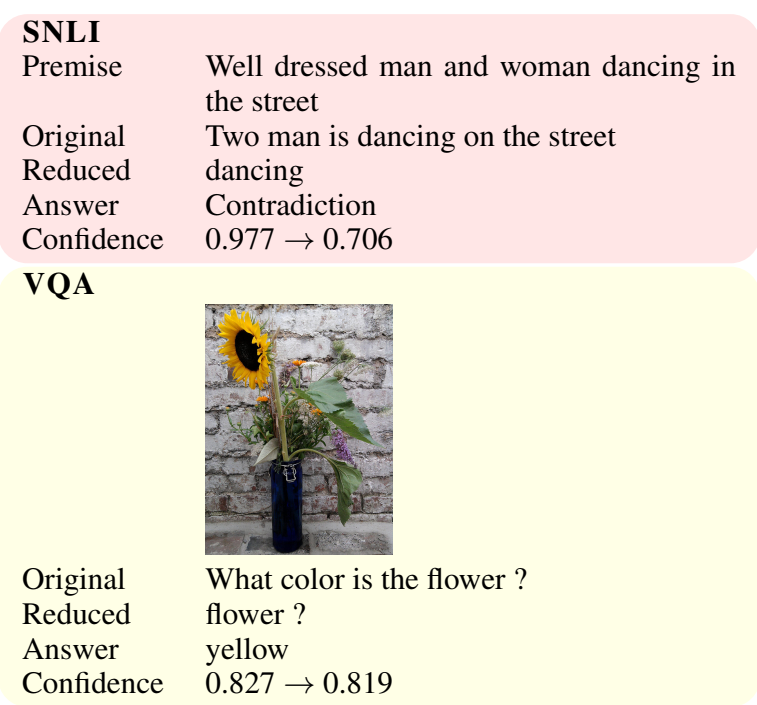

Figure 2: Examples of original and reduced inputs where the models predict the same Answer. Reduced shows the input after reduction. We remove words from the hypothesis for SNLI, questions for SQUAD and VQA. Given the nonsensical reduced inputs, humans would not be able to provide the answer with high confidence, yet, the neural models do.

With beam search, input reduction finds extremely short reduced examples with little to no decrease in the model's confidence on its original predictions. Figure 3 compares the length of input sentences before and after the reduction. For all three tasks, we can often reduce the sentence to only one word. Figure 4 compares the model's confidence on original and reduced inputs. On SQUAD and SNLI the confidence decreases slightly, and on VQA the confidence even increases.

\subsection{Humans Confused by Reduced Inputs}

On the reduced examples, the models retain their original predictions despite short input lengths. The following experiments examine whether these predictions are justified or pathological, based on how humans react to the reduced inputs.

For each task, we sample 200 examples that are correctly classified by the model and generate their reduced examples. In the first setting, we compare the human accuracy on original and reduced examples. We recruit two groups of crowd workers and task them with textual entailment, reading comprehension, or visual question answering. We show one group the original inputs and the other the reduced. Humans are no longer able to give 


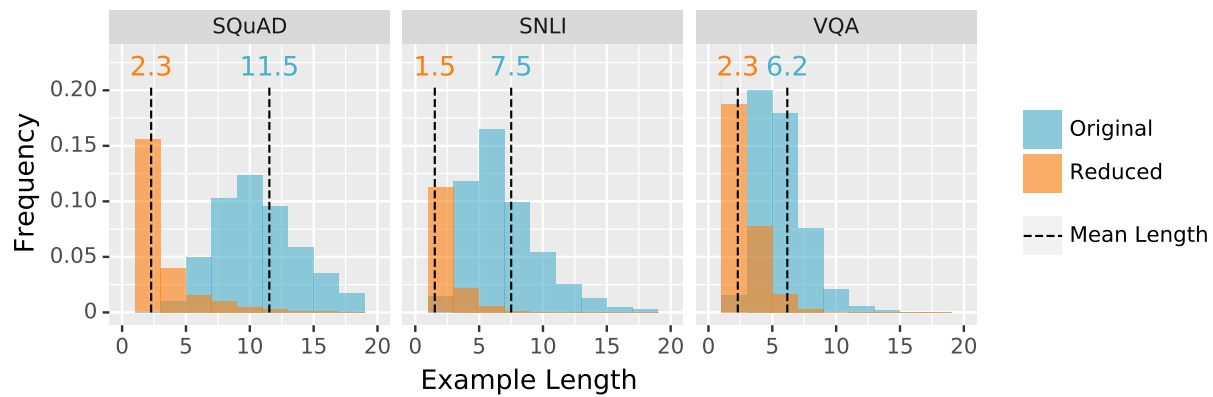

Figure 3: Distribution of input sentence length before and after reduction. For all three tasks, the input is often reduced to one or two words without changing the model's prediction.

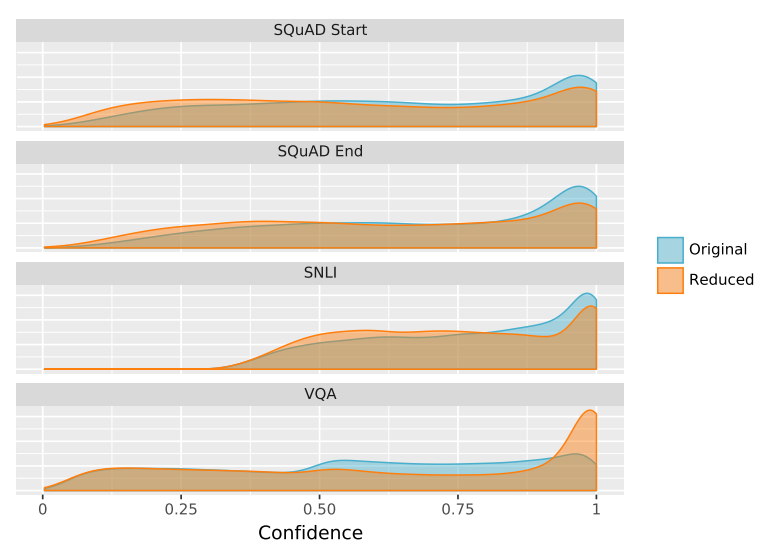

Figure 4: Density distribution of model confidence on reduced inputs is similar to the original confidence. In SQUAD, we predict the beginning and the end of the answer span, so we show the confidence for both.

the correct answer, showing a significant accuracy loss on all three tasks (compare Original and Reduced in Table 1).

The second setting examines how random the reduced examples appear to humans. For each of the original examples, we generate a version where words are randomly removed until the length matches the one generated by input reduction. We present the original example along with the two reduced examples and ask crowd workers their preference between the two reduced ones. The workers' choice is almost fifty-fifty (the vs. Random in Table 1): the reduced examples appear almost random to humans.

These results leave us with two puzzles: why are the models highly confident on the nonsensical reduced examples? And why, when the leave-oneout method selects important words that appear reasonable to humans, the input reduction process selects ones that are nonsensical?

\begin{tabular}{lccc} 
Dataset & Original & Reduced & vs. Random \\
\hline SQUAD & 80.58 & 31.72 & 53.70 \\
SNLI-E & 76.40 & 27.66 & 42.31 \\
SNLI-N & 55.40 & 52.66 & 50.64 \\
SNLI-C & 76.20 & 60.60 & 49.87 \\
VQA & 76.11 & 40.60 & 61.60
\end{tabular}

Table 1: Human accuracy on Reduced examples drops significantly compared to the Original examples, however, model predictions are identical. The reduced examples also appear random to humans-they do not prefer them over random inputs (vs. Random). For SQUAD, accuracy is reported using F1 scores, other numbers are percentages. For SNLI, we report results on the three classes separately: entailment $(-E)$, neutral $(-N)$, and contradiction $(-C)$.

\section{Making Sense of Reduced Inputs}

Having established the incongruity of our definition of importance vis-à-vis human judgements, we now investigate possible explanations for these results. We explain why model confidence can empower methods such as leave-one-out to generate reasonable interpretations but also lead to pathologies under input reduction. We attribute these results to two issues of neural models.

\subsection{Model Overconfidence}

Neural models are overconfident in their predictions (Guo et al., 2017). One explanation for overconfidence is overfitting: the model overfits the negative log-likelihood loss during training by learning to output low-entropy distributions over classes. Neural models are also overconfident on examples outside the training data distribution. As Goodfellow et al. (2015) observe for image classification, samples from pure noise can sometimes trigger highly confident predictions. These socalled rubbish examples are degenerate inputs that 
a human would trivially classify as not belonging to any class but for which the model predicts with high confidence. Goodfellow et al. (2015) argue that the rubbish examples exist for the same reason that adversarial examples do: the surprising linear nature of neural models. In short, the confidence of a neural model is not a robust estimate of its prediction uncertainty.

Our reduced inputs satisfy the definition of rubbish examples: humans have a hard time making predictions based on the reduced inputs (Table 1), but models make predictions with high confidence (Figure 4). Starting from a valid example, input reduction transforms it into a rubbish example.

The nonsensical, almost random results are best explained by looking at a complete reduction path (Figure 5). In this example, the transition from valid to rubbish happens immediately after the first step: following the removal of "Broncos", humans can no longer determine which team the question is asking about, but model confidence remains high. Not being able to lower its confidence on rubbish examples-as it is not trained to do sothe model neglects "Broncos" and eventually the process generates nonsensical results.

In this example, the leave-one-out method will not highlight "Broncos". However, this is not a failure of the interpretation method but of the model itself. The model assigns a low importance to "Broncos" in the first step, causing it to be removed-leave-one-out would be able to expose this particular issue by not highlighting "Broncos". However, in cases where a similar issue only appear after a few unimportant words are removed, the leave-one-out method would fail to expose the unreasonable model behavior.

Input reduction can expose deeper issues of model overconfidence and stress test a model's uncertainty estimation and interpretability.

\subsection{Second-order Sensitivity}

So far, we have seen that the output of a neural model is sensitive to small changes in its input. We call this first-order sensitivity, because interpretation based on input gradient is a first-order Taylor expansion of the model near the input (Simonyan et al., 2014). However, the interpretation also shifts drastically with small input changes (Figure 6). We call this second-order sensitivity.

The shifting heatmap suggests a mismatch between the model's first- and second-order sensi-
SQUAD

Context: The Panthers used the San Jose State practice facility and stayed at the San Jose Marriott. The Broncos practiced at Stanford University and stayed at the Santa Clara Marriott.

Question:

$(0.90,0.89)$ Where did the Broncos practice for the Super Bowl ?

$(0.92,0.88)$ Where did the practice for the Super Bowl ?

$(0.91,0.88)$ Where did practice for the Super Bowl ?

$(0.92,0.89)$ Where did practice the Super Bowl ?

$(0.94,0.90)$ Where did practice the Super ?

$(0.93,0.90)$ Where did practice Super ?

$(0.40,0.50)$ did practice Super ?

Figure 5: A reduction path for a SQUAD validation example. The model prediction is always correct and its confidence stays high (shown on the left in parentheses) throughout the reduction. Each line shows the input at that step with an underline indicating the word to remove next. The question becomes unanswerable immediately after "Broncos" is removed in the first step. However, in the context of the original question, "Broncos" is the least important word according to the input gradient.

tivities. The heatmap shifts when, with respect to the removed word, the model has low first-order sensitivity but high second-order sensitivity.

Similar issues complicate comparable interpretation methods for image classification models. For example, Ghorbani et al. (2017) modify image inputs so the highlighted features in the interpretation change while maintaining the same prediction. To achieve this, they iteratively modify the input to maximize changes in the distribution of feature importance. In contrast, the shifting heatmap we observe occurs by only removing the least impactful features without a targeted optimization. They also speculate that the steepest gradient direction for the first- and secondorder sensitivity values are generally orthogonal. Loosely speaking, the shifting heatmap suggests that the direction of the smallest gradient value can sometimes align with very steep changes in second-order sensitivity.

When explaining individual model predictions, the heatmap suggests that the prediction is made based on a weighted combination of words, as in a linear model, which is not true unless the model is indeed taking a weighted sum such as in a DAN (Iyyer et al., 2015). When the model composes representations by a non-linear combination of words, a linear interpretation oblivious to second-order sensitivity can be misleading. 
SQUAD

Context: QuickBooks sponsored a "Small Business Big Game" contest, in which Death Wish Coffee had a 30-second commercial aired free of charge courtesy of QuickBooks. Death Wish Coffee beat out nine other contenders from across the United States for the free advertisement.

Question:

What company won free advertisement due to QuickBooks contest ?

What company won free advertisement due to QuickBooks ?

What company won free advertisement due to ?

What company won free due to ?

What won free due to ?

What won due to ?

What won due to

What won due

What won

What

Figure 6: Heatmap generated with leave-one-out shifts drastically despite only removing the least important word (underlined) at each step. For instance, "advertisement", is the most important word in step two but becomes the least important in step three.

\section{Mitigating Model Pathologies}

The previous section explains the observed pathologies from the perspective of overconfidence: models are too certain on rubbish examples when they should not make any prediction. Human experiments in Section 2.3 confirm that the reduced examples fit the definition of rubbish examples. Hence, a natural way to mitigate the pathologies is to maximize model uncertainty on the reduced examples.

\subsection{Regularization on Reduced Inputs}

To maximize model uncertainty on reduced examples, we use the entropy of the output distribution as an objective. Given a model $f$ trained on a dataset $(\mathcal{X}, \mathcal{Y})$, we generate reduced examples using input reduction for all training examples $\mathcal{X}$. Beam search often yields multiple reduced versions with the same minimum length for each input $\mathbf{x}$, and we collect all of these versions together to form $\tilde{\mathcal{X}}$ as the "negative" example set.

Let $\mathbb{H}(\cdot)$ denote the entropy and $f(y \mid \mathbf{x})$ denote the probability of the model predicting $y$ given $\mathbf{x}$. We fine-tune the existing model to simultaneously maximize the log-likelihood on regular examples and the entropy on reduced examples:

$$
\sum_{(\mathbf{x}, y) \in(\mathcal{X}, \mathcal{Y})} \log (f(y \mid \mathbf{x}))+\lambda \sum_{\tilde{\mathbf{x}} \in \tilde{\mathcal{X}}} \mathbb{H}(f(y \mid \tilde{\mathbf{x}})),
$$

where hyperparameter $\lambda$ controls the trade-off between the two terms. Similar entropy regularization is used by Pereyra et al. (2017), but not in

\begin{tabular}{lcccccc} 
& \multicolumn{2}{c}{ Accuracy } & & \multicolumn{2}{c}{ Reduced length } \\
\cline { 2 - 3 } \cline { 6 - 6 } & Before & After & & Before & After \\
\hline SQUAD & 77.41 & 78.03 & & 2.27 & 4.97 \\
SNLI & 85.71 & 85.72 & & 1.50 & 2.20 \\
VQA & 61.61 & 61.54 & & 2.30 & 2.87
\end{tabular}

Table 2: Model Accuracy on regular validation examples remains largely unchanged after fine-tuning. However, the length of the reduced examples (Reduced length) increases on all three tasks, making them less likely to appear nonsensical to humans.

combination with input reduction; their entropy term is calculated on regular examples rather than reduced examples.

\subsection{Regularization Mitigates Pathologies}

On regular examples, entropy regularization does no harm to model accuracy, with a slight increase for SQUAD (Accuracy in Table 2).

After entropy regularization, input reduction produces more reasonable reduced inputs (Figure 7). In the SQUAD example from Figure 1, the reduced question changed from "did" to "spend Astor money on ?" after fine-tuning. The average length of reduced examples also increases across all tasks (Reduced length in Table 2). To verify that model overconfidence is indeed mitigatedthat the reduced examples are less "rubbish" compared to before fine-tuning - we repeat the human experiments from Section 2.3.

Human accuracy increases across all three tasks (Table 3). We also repeat the vs. Random experiment: we re-generate the random examples to match the lengths of the new reduced examples from input reduction, and find humans now prefer the reduced examples to random ones. The increase in both human performance and preference suggests that the reduced examples are more reasonable; model pathologies have been mitigated.

While these results are promising, it is not clear whether our input reduction method is necessary to achieve them. To provide a baseline, we finetune models using inputs randomly reduced to the same lengths as the ones generated by input reduction. This baseline improves neither the model accuracy on regular examples nor interpretability under input reduction (judged by lengths of reduced examples). Input reduction is effective in generating negative examples to counter model overconfidence. 


$\begin{array}{ll}\text { SQUAD } & \\ \text { Context } & \begin{array}{l}\text { In } 1899, \text { John Jacob Astor IV invested } \\ \$ 100,000 \text { for Tesla to further develop } \\ \text { and produce a new lighting system. In- } \\ \text { stead, Tesla used the money to fund his } \\ \text { Colorado Springs experiments. }\end{array} \\ \begin{array}{ll}\text { Original } & \text { What did Tesla spend Astor's money on ? } \\ \text { Answer } & \text { Colorado Springs experiments } \\ \text { Before } & \text { did } \\ \text { After } & \text { spend Astor money on ? } \\ \text { Confidence } & 0.78 \rightarrow 0.91 \rightarrow 0.52 \\ \text { SNLI } & \\ \text { Premise } & \text { Well dressed man and woman dancing in } \\ & \text { the street } \\ \text { Original } & \text { Two man is dancing on the street } \\ \text { Answer } & \text { Contradiction } \\ \text { Before } & \text { dancing } \\ \text { After } & \text { two man dancing } \\ \text { Confidence } & 0.977 \rightarrow 0.706 \rightarrow 0.717 \\ \text { VQA } & \\ \text { Original } & \text { What color is the flower? } \\ \text { Answer } & \text { yellow } \\ \text { Before } & \text { flower ? } \\ \text { After } & \text { What color is flower ? } \\ \text { Confidence } & 0.847 \rightarrow 0.918 \rightarrow 0.745\end{array} \\ \end{array}$

Figure 7: SQUAD example from Figure 1, SNLI and VQA (image omitted) examples from Figure 2. We apply input reduction to models both Before and After entropy regularization. The models still predict the same Answer, but the reduced examples after fine-tuning appear more reasonable to humans.

\section{Discussion}

Rubbish examples have been studied in the image domain (Goodfellow et al., 2015; Nguyen et al., 2015), but to our knowledge not for NLP. Our input reduction process gradually transforms a valid input into a rubbish example. We can often determine which word's removal causes the transition to occur-for example, removing "Broncos" in Figure 5. These rubbish examples are particularly interesting, as they are also adversarial: the difference from a valid example is small, unlike image rubbish examples generated from pure noise which are far outside the training data distribution.

The robustness of NLP models has been studied extensively (Papernot et al., 2016; Jia and Liang, 2017; Iyyer et al., 2018; Ribeiro et al., 2018), and most studies define adversarial examples similar to the image domain: small perturbations to the input lead to large changes in the output. HotFlip (Ebrahimi et al., 2017) uses a gradient-based approach, similar to image adversarial examples, to flip the model prediction by perturbing a few characters or words. Our work and Belinkov and Bisk (2018) both identify cases where noisy

\begin{tabular}{lcclll} 
& \multicolumn{2}{c}{ Accuracy } & & \multicolumn{2}{c}{ vs. Random } \\
\cline { 2 - 3 } \cline { 6 - 7 } & Before & After & & Before & After \\
\hline SQUAD & 31.72 & 51.61 & & 53.70 & 62.75 \\
SNLI-E & 27.66 & 32.37 & & 42.31 & 50.62 \\
SNLI-N & 52.66 & 50.50 & & 50.64 & 58.94 \\
SNLI-C & 60.60 & 63.90 & & 49.87 & 56.92 \\
VQA & 40.60 & 51.85 & & 61.60 & 61.88
\end{tabular}

Table 3: Human Accuracy increases after fine-tuning the models. Humans also prefer gradient-based reduced examples over randomly reduced ones, indicating that the reduced examples are more meaningful to humans after regularization.

user inputs become adversarial by accident: common misspellings break neural machine translation models; we show that incomplete user input can lead to unreasonably high model confidence.

Other failures of interpretation methods have been explored in the image domain. The sensitivity issue of gradient-based interpretation methods, similar to our shifting heatmaps, are observed by Ghorbani et al. (2017) and Kindermans et al. (2017). They show that various forms of input perturbation-from adversarial changes to simple constant shifts in the image input-cause significant changes in the interpretation. Ghorbani et al. (2017) make a similar observation about secondorder sensitivity, that "the fragility of interpretation is orthogonal to fragility of the prediction".

Previous work studies biases in the annotation process that lead to datasets easier than desired or expected which eventually induce pathological models. We attribute our observed pathologies primarily to the lack of accurate uncertainty estimates in neural models trained with maximum likelihood. SNLI hypotheses contain artifacts that allow training a model without the premises (Gururangan et al., 2018); we apply input reduction at test time to the hypothesis. Similarly, VQA images are surprisingly unimportant for training a model; we reduce the question. The recent SQUAD 2.0 (Rajpurkar et al., 2018) augments the original reading comprehension task with an uncertainty modeling requirement, the goal being to make the task more realistic and challenging.

Section 3.1 explains the pathologies from the overconfidence perspective. One explanation for overconfidence is overfitting: Guo et al. (2017) show that, late in maximum likelihood training, 
the model learns to minimize loss by outputting low-entropy distributions without improving validation accuracy. To examine if overfitting can explain the input reduction results, we run input reduction using DRQA model checkpoints from every training epoch. Input reduction still achieves similar results on earlier checkpoints, suggesting that better convergence in maximum likelihood training cannot fix the issues by itself-we need new training objectives with uncertainty estimation in mind.

\subsection{Methods for Mitigating Pathologies}

We use the reduced examples generated by input reduction to regularize the model and improve its interpretability. This resembles adversarial training (Goodfellow et al., 2015), where adversarial examples are added to the training set to improve model robustness. The objectives are different: entropy regularization encourages high uncertainty on rubbish examples, while adversarial training makes the model less sensitive to adversarial perturbations.

Pereyra et al. (2017) apply entropy regularization on regular examples from the start of training to improve model generalization. A similar method is label smoothing (Szegedy et al., 2016). In comparison, we fine-tune a model with entropy regularization on the reduced examples for better uncertainty estimates and interpretations.

To mitigate overconfidence, Guo et al. (2017) propose post-hoc fine-tuning a model's confidence with Platt scaling. This method adjusts the softmax function's temperature parameter using a small held-out dataset to align confidence with accuracy. However, because the output is calibrated using the entire confidence distribution, not individual values, this does not reduce overconfidence on specific inputs, such as the reduced examples.

\subsection{Generalizability of Findings}

To highlight the erratic model predictions on short examples and provide a more intuitive demonstration, we present paired-input tasks. On these tasks, the short lengths of reduced questions and hypotheses obviously contradict the necessary number of words for a human prediction (further supported by our human studies). We also apply input reduction to single-input tasks including sentiment analysis (Maas et al., 2011) and Quizbowl (BoydGraber et al., 2012), achieving similar results.
Interestingly, the reduced examples transfer to other architectures. In particular, when we feed fifty reduced SNLI inputs from each class-generated with the BIMPM model (Wang et al., 2017) - through the Decomposable Attention Model (Parikh et al., 2016), ${ }^{2}$ the same prediction is triggered $81.3 \%$ of the time.

\section{Conclusion}

We introduce input reduction, a process that iteratively removes unimportant words from an input while maintaining a model's prediction. Combined with gradient-based importance estimates often used for interpretations, we expose pathological behaviors of neural models. Without lowering model confidence on its original prediction, an input sentence can be reduced to the point where it appears nonsensical, often consisting of one or two words. Human accuracy degrades when shown the reduced examples instead of the original, in contrast to neural models which maintain their original predictions.

We explain these pathologies with known issues of neural models: overconfidence and sensitivity to small input changes. The nonsensical reduced examples are caused by inaccurate uncertainty estimates - the model is not able to lower its confidence on inputs that do not belong to any label. The second-order sensitivity is another issue why gradient-based interpretation methods may fail to align with human perception: a small change in the input can cause, at the same time, a minor change in the prediction but a large change in the interpretation. Input reduction perturbs the input multiple times and can expose deeper issues of model overconfidence and oversensitivity that other methods cannot. Therefore, it can be used to stress test the interpretability of a model.

Finally, we fine-tune the models by maximizing entropy on reduced examples to mitigate the deficiencies. This improves interpretability without sacrificing model accuracy on regular examples.

To properly interpret neural models, it is important to understand their fundamental characteristics: the nature of their decision surfaces, robustness against adversaries, and limitations of their training objectives. We explain fundamental difficulties of interpretation due to pathologies in neural models trained with maximum likelihood. Our

\footnotetext{
${ }^{2}$ http://demo.allennlp.org/ textual-entailment
} 
work suggests several future directions to improve interpretability: more thorough evaluation of interpretation methods, better uncertainty and confidence estimates, and interpretation beyond bagof-word heatmap.

\section{Acknowledgments}

Feng was supported under subcontract to Raytheon BBN Technologies by DARPA award HR0011-15-C-0113. JBG is supported by NSF Grant IIS1652666. Any opinions, findings, conclusions, or recommendations expressed here are those of the authors and do not necessarily reflect the view of the sponsor. The authors would like to thank Hal Daumé III, Alexander M. Rush, Nicolas Papernot, members of the CLIP lab at the University of Maryland, and the anonymous reviewers for their feedback.

\section{References}

Stanislaw Antol, Aishwarya Agrawal, Jiasen Lu, Margaret Mitchell, Dhruv Batra, C. Lawrence Zitnick, and Devi Parikh. 2015. VQA: Visual question answering. In International Conference on Computer Vision.

Leila Arras, Franziska Horn, Grégoire Montavon, Klaus-Robert Müller, and Wojciech Samek. 2016. Explaining predictions of non-linear classifiers in NLP. In Workshop on Representation Learning for $N L P$.

David Baehrens, Timon Schroeter, Stefan Harmeling, Motoaki Kawanabe, Katja Hansen, and KlausRobert Müller. 2010. How to explain individual classification decisions. Journal of Machine Learning Research.

Yonatan Belinkov and Yonatan Bisk. 2018. Synthetic and natural noise both break neural machine translation. In Proceedings of the International Conference on Learning Representations.

Samuel R. Bowman, Gabor Angeli, Christopher Potts, and Christopher D. Manning. 2015. A large annotated corpus for learning natural language inference. In Proceedings of Empirical Methods in Natural Language Processing.

Jordan L. Boyd-Graber, Brianna Satinoff, He He, and Hal Daumé III. 2012. Besting the quiz master: Crowdsourcing incremental classification games. In Proceedings of Empirical Methods in Natural Language Processing.

Danqi Chen, Adam Fisch, Jason Weston, and Antoine Bordes. 2017. Reading wikipedia to answer opendomain questions. In Proceedings of the Association for Computational Linguistics.
Javid Ebrahimi, Anyi Rao, Daniel Lowd, and Dejing Dou. 2017. HotFlip: White-box adversarial examples for text classification. In Proceedings of the Association for Computational Linguistics.

Amirata Ghorbani, Abubakar Abid, and James Y. Zou. 2017. Interpretation of neural networks is fragile. arXiv preprint arXiv: 1710.10547.

Ian J. Goodfellow, Jonathon Shlens, and Christian Szegedy. 2015. Explaining and harnessing adversarial examples. In Proceedings of the International Conference on Learning Representations.

Chuan Guo, Geoff Pleiss, Yu Sun, and Kilian Q. Weinberger. 2017. On calibration of modern neural networks. In Proceedings of the International Conference of Machine Learning.

Suchin Gururangan, Swabha Swayamdipta, Omer Levy, Roy Schwartz, Samuel R. Bowman, and Noah A. Smith. 2018. Annotation artifacts in natural language inference data. In Conference of the North American Chapter of the Association for Computational Linguistics.

Mohit Iyyer, Varun Manjunatha, Jordan Boyd-Graber, and Hal Daumé III. 2015. Deep unordered composition rivals syntactic methods for text classification. In Proceedings of the Association for Computational Linguistics.

Mohit Iyyer, John Wieting, Kevin Gimpel, and Luke S. Zettlemoyer. 2018. Adversarial example generation with syntactically controlled paraphrase networks. In Conference of the North American Chapter of the Association for Computational Linguistics.

Robin Jia and Percy Liang. 2017. Adversarial examples for evaluating reading comprehension systems. In Proceedings of Empirical Methods in Natural Language Processing.

Vahid Kazemi and Ali Elqursh. 2017. Show, ask, attend, and answer: A strong baseline for visual question answering. arXiv preprint arXiv: 1704.03162.

Pieter-Jan Kindermans, Sara Hooker, Julius Adebayo, Maximilian Alber, Kristof T. Schütt, Sven Dähne, Dumitru Erhan, and Been Kim. 2017. The (un)reliability of saliency methods. arXiv preprint arXiv: 1711.00867.

Jiwei Li, Xinlei Chen, Eduard H. Hovy, and Daniel Jurafsky. 2016a. Visualizing and understanding neural models in NLP. In Conference of the North American Chapter of the Association for Computational Linguistics.

Jiwei Li, Will Monroe, and Daniel Jurafsky. 2016b. Understanding neural networks through representation erasure. arXiv preprint arXiv: 1612.08220.

Andrew Maas, Raymond Daly, Peter Pham, Dan Huang, Andrew Ng, and Christopher Potts. 2011. Learning word vectors for sentiment analysis. In 
Proceedings of the Association for Computational Linguistics.

W. James Murdoch, Peter J. Liu, and Bin Yu. 2018. Beyond word importance: Contextual decomposition to extract interactions from lstms. In Proceedings of the International Conference on Learning Representations.

Anh Mai Nguyen, Jason Yosinski, and Jeff Clune. 2015. Deep neural networks are easily fooled: High confidence predictions for unrecognizable images. In Computer Vision and Pattern Recognition.

Nicolas Papernot, Patrick D. McDaniel, Ananthram Swami, and Richard E. Harang. 2016. Crafting adversarial input sequences for recurrent neural networks. IEEE Military Communications Conference.

Ankur P. Parikh, Oscar Täckström, Dipanjan Das, and Jakob Uszkoreit. 2016. A decomposable attention model for natural language inference. In Proceedings of Empirical Methods in Natural Language Processing.

Gabriel Pereyra, George Tucker, Jan Chorowski, Lukasz Kaiser, and Geoffrey E. Hinton. 2017. Regularizing neural networks by penalizing confident output distributions. In Proceedings of the International Conference on Learning Representations.

Pranav Rajpurkar, Robin Jia, and Percy Liang. 2018. Know what you don't know: Unanswerable questions for squad. In Proceedings of the Association for Computational Linguistics.

Pranav Rajpurkar, Jian Zhang, Konstantin Lopyrev, and Percy Liang. 2016. SQuAD: 100, 000+ questions for machine comprehension of text. In Proceedings of Empirical Methods in Natural Language Processing.

Marco Túlio Ribeiro, Sameer Singh, and Carlos Guestrin. 2016. Why should i trust you?": Explaining the predictions of any classifier. In Knowledge Discovery and Data Mining.

Marco Tulio Ribeiro, Sameer Singh, and Carlos Guestrin. 2018. Semantically equivalent adversarial rules for debugging nlp models. In Proceedings of the Association for Computational Linguistics.

Karen Simonyan, Andrea Vedaldi, and Andrew Zisserman. 2014. Deep inside convolutional networks: Visualising image classification models and saliency maps. In Proceedings of the International Conference on Learning Representations.

Christian Szegedy, Vincent Vanhoucke, Sergey Ioffe, Jon Shlens, and Zbigniew Wojna. 2016. Rethinking the inception architecture for computer vision. In Computer Vision and Pattern Recognition.

Christian Szegedy, Wojciech Zaremba, Ilya Sutskever, Joan Bruna, Dumitru Erhan, Ian J. Goodfellow, and Rob Fergus. 2014. Intriguing properties of neural networks. In Proceedings of the International Conference on Learning Representations.

Zhiguo Wang, Wael Hamza, and Radu Florian. 2017. Bilateral multi-perspective matching for natural language sentences. In International Joint Conference on Artificial Intelligence. 